\title{
Nonlinear Response of Graphite-Epoxy Composite Thin-walled structure under Elevated Thermal Environment
}

\author{
Yundong Sha, a , Fei $\mathrm{Xu}^{2, \mathrm{~b}}$ and Zhijun $\mathrm{Gao}^{3, \mathrm{c}}$ \\ * Shenyang Aerospace University, Liaoning Key Laboratory of Digital Technology Simulation and \\ Test Techniques, Liaoning, Shenyang, 110136, P.R. China
}

ydsha2003@vip.sina.com

\section{Keywords: Thin-walled structure, Composite material, Nonlinear response, Post-buckling}

\begin{abstract}
Composite materials thin-walled structures are widely used as skin panel in flight vehicles in recent years. These structures will encounter severe complex loading conditions, which may be a combination of mechanical, aerodynamic, thermal and acoustic loads. Thin-walled structures subjected to this kind of loadings will exhibit nonlinear response; as a result, fatigue failure will occur. High temperature may cause large thermal deflection and stress, for some special conditions, may cause thermal buckling. Once the thermal buckling appears, the stiffness will change correspondingly, it will cause significant influence on the dynamic response and fatigue failure. Accordingly, it is important to research the nonlinear response of this kind of structures under elevated thermal environment.

Nonlinear response and thermal pre-buckling/post-buckling behavior of a Graphite-Epoxy composite plate subjected to server thermal loading is numerically investigated in this paper. A composite laminated plate with clamped-clamped boundary conditions is chosen as simulated body, nonlinear finite element model is developed using the first-order shear deformable plate theory, Von Karman strain-displacement relations, and the principle of virtual work. The thermal load is assumed to be a steady-state with different predefined temperature distribution. The thermal strain is stated as an integral quantity of the thermal expansion coefficient with respect to temperature. Then the modes of the plate are analyzed, the nature frequencies and modal shapes are obtained. The critical temperature of buckling is calculated. The static nonlinear equations of motions are solved by the Newton-Raphson iteration technique to obtain the thermal post-buckling deflection. The Riks method is used to analyze static post-buckling behavior. In the numerical examples, four types of situations are studied, which include i) the buckling behaviors for different initial imperfections, ii) the buckling behaviors for different thickness to width ratios, and iii) The buckling behaviors for different width to length ratios; The critical temperature, the static thermal post-buckling deflection and the load to displacement relation are presented respectively.

The influences of different boundary conditions on the buckling behaviors of the plate are achieved as well. The simulation method and results presented in this paper can be valuable references for further analysis of the nonlinear responses of thin-walled structures under complex loading conditions.
\end{abstract}

\section{Introduction}

Composite materials thin-walled structures are widely used as skin panel in flight vehicles in recent years. These structures will encounter severe complex loading conditions, which may be a combination of mechanical, aerodynamic, thermal and acoustic loads. Thin-walled structures subjected to this kind of loadings will exhibit nonlinear response; as a result, fatigue failure will occur. High temperature may cause large thermal deflection and stress, for some special conditions, may cause thermal buckling. Once the thermal buckling appears, the stiffness will change correspondingly, it will cause significant influence on the dynamic response and fatigue failure. Accordingly, it is important to research the nonlinear response of this kind of structures under elevated Thermal environment. 
Nonlinear response and thermal pre-buckling/post-buckling behaviors of a Graphite-Epoxy composite plate subjected to severer thermal loading is numerical investigated in this paper. A composite laminated plate with clamped-clamped boundary conditions is chosen as simulated body, nonlinear finite element model is developed using the first-order shear deformable plate theory, Von Karman strain-displacement relations, and the principle of virtual work. The thermal load is assumed to be a steady-state with different predefined temperature distribution. The thermal strain is stated as an integral quantity of the thermal expansion coefficient with respect to temperature. Then the modes of the plate are analyzed, the nature frequencies and modal shapes are obtained. The critical temperature of buckling is calculated. The static nonlinear equations of motions are solved by the Newton-Raphson iteration technique to obtain the thermal post-buckling deflection. The Riks method is used to analyze static post-buckling behavior. In the numerical examples, five types of situations are studied, which include; i) the buckling behaviors for different initial imperfections, ii) the buckling behaviors for different thickness to width ratios, and iii) The buckling behaviors for different width to length ratios; iv) The buckling behaviors for different boundary conditions. The critical temperature and the load-displacement relation are presented respectively. The simulation method and results presented in this paper can be valuable references for further analysis of the nonlinear responses of thin-walled structures under complex loading conditions.

A rectangular Graphite-Epoxy plate of eight layers $[0 / 40 /-45 / 90]_{\mathrm{s}}$ is investigated in this paper, the material properties the of the plate are show in Table 1

Table 1. Material properties of the composite materials plate

\begin{tabular}{cccccccc}
\hline $\begin{array}{c}E_{2} \\
{[\mathrm{GPa}]}\end{array}$ & $\begin{array}{c}E_{2} \\
{[\mathrm{GPa}]}\end{array}$ & $\begin{array}{c}G_{12}=G_{13} \\
{[\mathrm{GPa}]}\end{array}$ & $\begin{array}{c}G_{13} \\
{[\mathrm{GPa}]}\end{array}$ & $\begin{array}{c}\rho \\
{\left[\mathrm{kg} / \mathrm{m}^{3}\right]}\end{array}$ & $v$ & $\begin{array}{c}\alpha_{1} \\
{\left[\Delta T /{ }^{\circ} \mathrm{C}\right]}\end{array}$ & $\begin{array}{c}\alpha_{2} \\
{\left[\Delta T /{ }^{\circ} C\right]}\end{array}$ \\
\hline 115 & 8.7 & 4.55 & 2.175 & 1430 & 0.22 & $8.5 \times 10^{-5}$ & $30.1 \times 10^{-6}$ \\
\hline
\end{tabular}

A proportional damping ratio with $\xi=0.01$ is used. The plate is modeled with a 6 by 6 mesh quarter plate with S8R element type which means 8 nodes of Hexahedral with linear reduced integration shell element.

\section{Governing Equations:}

The governing equations for a Graphite-Epoxy plate under the combined action of thermal loads can be stated as

$$
\left([K]-\left[K_{T}\right]+\left[K_{r}\right]+\frac{1}{2}\left[N_{1}\right]+\frac{1}{3}\left[N_{2}\right]\right)\{W\}=\left\{P_{T}\right\}-\left\{P_{r}\right\}
$$

Where $[\mathrm{K}]\left[\mathrm{K}_{\mathrm{T}}\right]$ and $\left[\mathrm{K}_{\mathrm{r}}\right]$ are the linear, thermal, and recovery-stress stiffness matrices respectively; $\left[\mathrm{n}_{1}\right]$ and $\left[\mathrm{n}_{2}\right]$ are the first and second-order nonlinear stiffness matrices; $\mathrm{K}$ is a shear correction factor; and $\left\{\mathrm{P}_{\mathrm{T}}\right\}$ are the thermal and $\left\{\mathrm{P}_{\mathrm{r}}\right\}$ is the recovery-stress vectors.

Solution Procedures: For the static thermal buckling problem, Eq. (1) reduces to

$$
\left([K]-\left[K_{T}\right]+\left[K_{r}\right]+\frac{1}{2}\left[N_{1}\right]+\frac{1}{3}\left[N_{2}\right]\right)\{W\}=\left\{P_{T}\right\}-\left\{P_{r}\right\}
$$

Introduce the function $\{\Psi(W)\}$ to Eq. (2).

$$
\{\Psi(W)\}=\left([K]-\left[K_{T}\right]+\left[K_{r}\right]+\frac{1}{2}\left[N_{1}\right]+\frac{1}{3}\left[N_{2}\right]\right)\{W\}-\left\{P_{T}\right\}+\left\{P_{r}\right\}=0
$$

Equation can be written in the form of a truncated Taylor series expansion as

$$
\{\Psi(W+\delta W)\}=\{\Psi(W)\}+\frac{d\{\Psi(W)\}}{d(W)}\{\delta W\} \cong 0
$$

Where 


$$
\frac{d\{\Psi(W)\}}{d(W)}=\left([K]-\left[K_{T}\right]+\left[K_{r}\right]+\left[N_{1}\right]+\left[N_{2}\right]\right)=\left[K_{\text {tan }}\right]
$$

Thus, the Newton-Raphson iteration procedure for the determination of the thermal post buckling deflections can be expressed as follows

$$
\begin{aligned}
& \{\Psi(W)\}_{i}=\left([K]-\left[K_{T}\right]+\left[K_{r}\right]+\frac{1}{2}\left(\left[N_{1}\right]\right)+\frac{1}{3}\left(\left[N_{2}\right]\right)_{i}\right)\{W\}-\left\{P_{T}\right\}+\left\{P_{r}\right\} \\
& {\left[K_{\tan }\right]_{i}\{\delta W\}_{i+1}=-\{\Psi(W)\}_{i}} \\
& \{\delta W\}_{i+1}=-\left[K_{\tan }\right]^{-1}\{\Psi(W)\}_{i} \\
& \{W\}_{i+1}=-\{W\}_{i}+\{\delta W\}_{i+1}
\end{aligned}
$$

Convergence occurs in the preceding procedure when the maximum value of $\{\delta W\}_{i+1}$ becomes less than a given tolerance $\varepsilon_{\text {tol }}\left(\right.$ i.e., $\left.\max \left|\{\delta W\}_{i+1}\right| \leq \varepsilon_{\text {tol }}\right)$.

\section{Numerical Results and Discussions}

Numerical analyses for the thermal buckling of a laminated composite-plate panel with five different situations.

For $1 \%, 5 \%, 10 \%, 50 \%, 70 \%, 85 \%, 100 \%, 150 \%$ five different ratio of initial imperfection to thickness situations, the LPF (the displacement-load proportional factor)-displacement curve are given in Fig.1. From the Fig.1 which $\mathrm{x}$ axis stands for the displacement of middle point of the panel and the $y$ axis means the full model load proportion factors, The influences of initial imperfection to plate's buckling behaviors are relatively smallest when the ratio of initial imperfection to the plate thickness equal to $50 \%$. The buckling behaviors of the plate is nonlinear when the ratio less than $50 \%$, and the buckling behaviors changes to linear when the ratio more than $50 \%$.

For five different ratios of thickness to width situations, the LPF-displacement curve can be expressed as Fig.2 and the nature frequency show in Table.2. Table.1 shows the nature frequency increase greatly with the thickness to width ratio increase, which illustrate the critical buckling temperature influenced by the ratio obviously. In Fig.2 the LPF-displacement curves become smoothly with the ratio of thickness to width increase, which mean clearly the stiffness of panel can be influenced by the ratio of thickness to width. Fig. 3 shows with the ratio of width to length increase, the critical temperature decrease gradually with the ratio goes up, but the Load-displacement curve in Fig. 4 does not change accordingly with this ratio. This illustrate that the composite plate's buckling behaviors do not affected by just change the ratio of width to length.

The character of critical temperature and buckling behaviors are show in Fig 5 and Fig6 for the two side simply supported, two side clamped-clamped, simply supported, clamped-clamped supported boundary conditions respectively.

Fig.5 and Fig6 show the clamped-clamped boundary condition can increase the critical temperature obviously and complete simply supported boundary condition is difficult to buckle than the two sides simply supported boundary condition. The boundary condition can't influence the buckling behaviors of the plate.

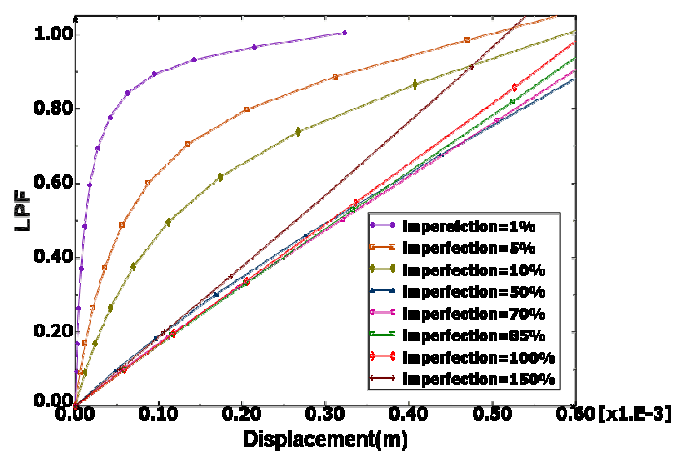

Fig. 1. LPF-Displacement curve for initial imperfection panel

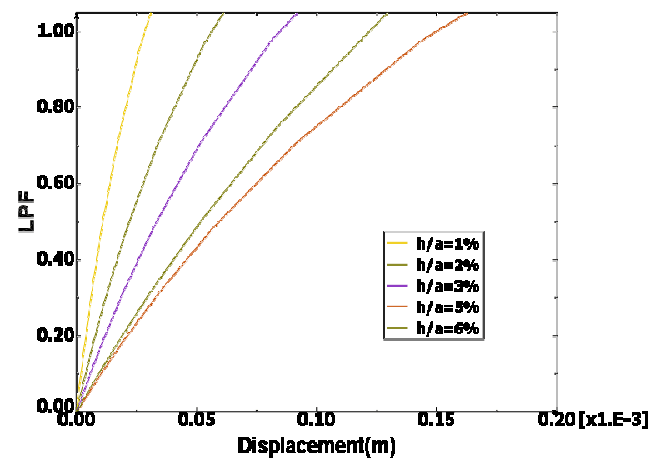

Fig. 2. LPF-Displacement curve for different thickness panel 
Table 2 Nature frequency of different thickness panel

\begin{tabular}{rccccc}
\hline $\begin{array}{c}\text { Modal } \\
\text { Number }\end{array}$ & $1 \%$ & $2 \%$ & $3 \%$ & $5 \%$ & $6 \%$ \\
\hline 1 & 57.114 & 221.74 & 479.86 & 1212.8 & 1655.6 \\
2 & 127.72 & 490.53 & 1042.1 & 2494.1 & 3294.6 \\
3 & 163.22 & 625.09 & 1319 & 3083.6 & 4014.4 \\
4 & 230.68 & 865.08 & 1777.6 & 3932.1 & 4991.4 \\
5 & 265.95 & 999.42 & 2049.7 & 4465.5 & 5608 \\
6 & 361.65 & 1324.7 & 2633.4 & 5404.7 & 6622.2 \\
\hline
\end{tabular}

3. For the critical temperature and buckling behaviors under different ratio of width to length, the result is show in Fig.3 and Fig.4.

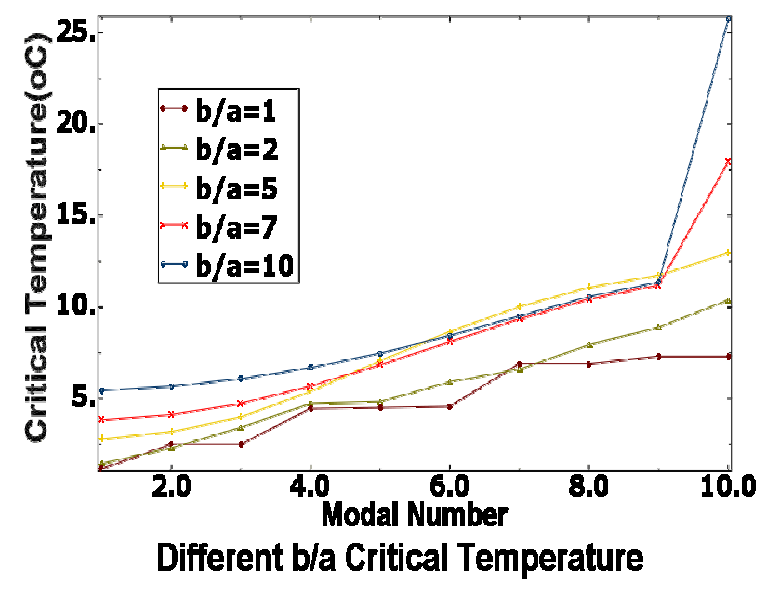

Fig. 3. Critical temperature for different $b / a$ pane

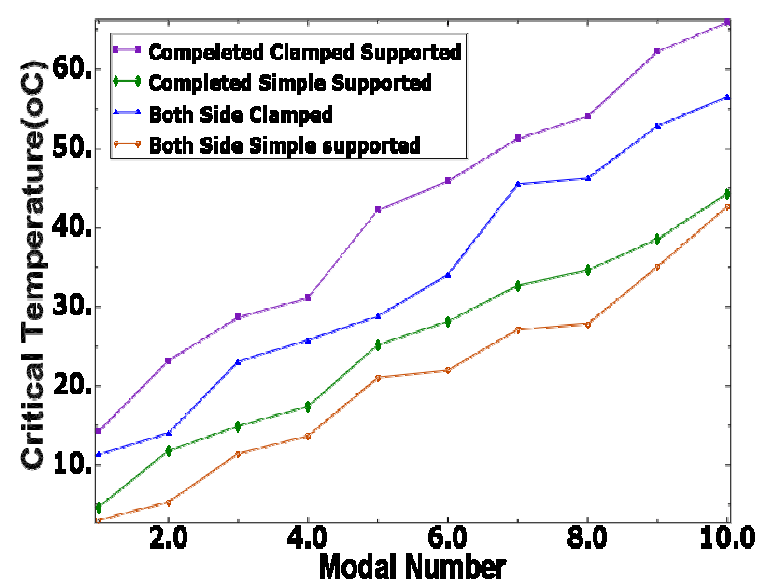

Fig. 5. Critical temperature for different boundary condition panel

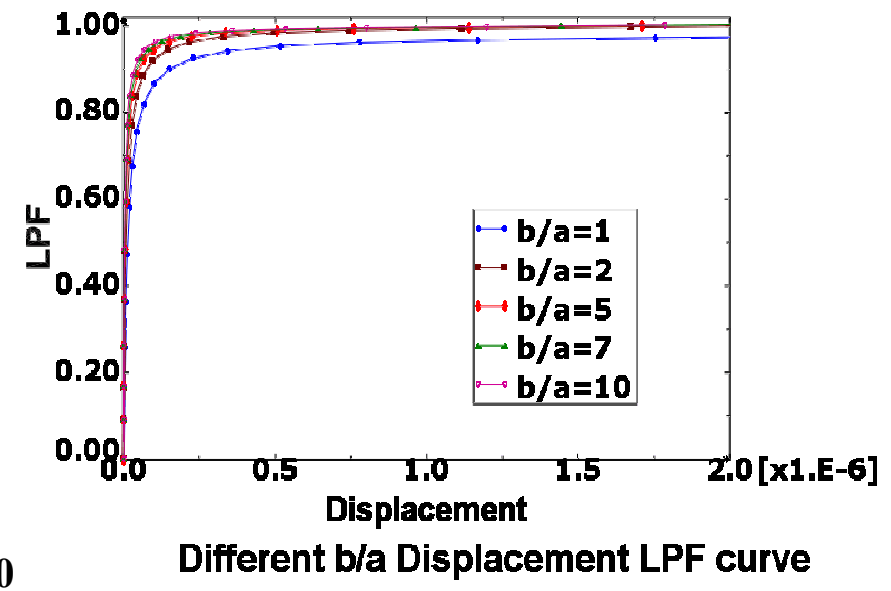

Fig. 4. LPF-Displacement curve for different b/a panel

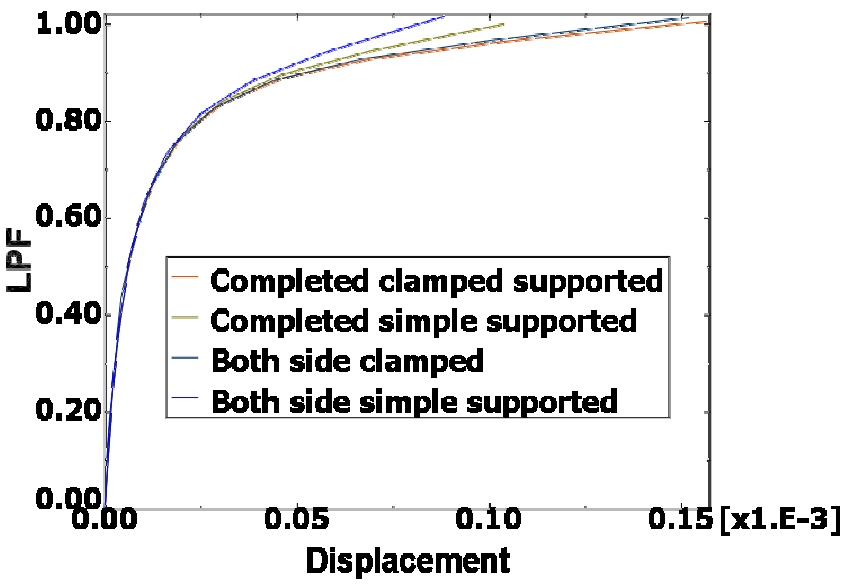

Fig. 6. LPF-Displacement curve for different boundary condition panel

\section{Conclusions}

(1) The influences of initial imperfection to plate's buckling behaviors are relatively smallest when the ratio of initial imperfection to the plate thickness equal to $50 \%$. The buckling behaviors of the plate is nonlinear when the ratio less than $50 \%$, and the buckling behaviors changes to linear when the ratio more than $50 \%$, which means that the stiffness of the plate become constant with the initial imperfection increase. 
(2) The critical buckling temperature increase greatly with the increase of the thickness to width ratio. The buckling behaviors become more linear with the ratio goes up, which can illustrate the stiffness of the plate, are influenced by the ratio obviously.

(3) The critical buckling temperature of plate decrease with the increase of the width to length ratio, but the buckling behaviors do not change, which mean that plates with smaller width to length ratio are more likely to buckle.

(4) The critical buckling temperature will greatly increase with clamped-clamped boundary conditions compared to the other boundary conditions with different combination of simply supported and clamped supported boundaries, but have less influence on the LPF-displacement curves of the plate.

\section{References}

[1] E. H Dowell., A Review of the Aero elastic Stability of Plates and Shells, AIAA Journal, Vol. 8, No. 3, (1970), pp. 385-399

[2] C. Mei., A Finite Element Formulation for the Large Deflection Random Response of Thermally Buckled Plates, AlAA 12th Aero acoustics Conference (1989)

[3] E. Riks., An incremental approach to the solution of snapping and buckling problems (1979)

[4] C. Charles., Finite element analysis of thermal post-buckling and vibrations of thermally buckled composite plates (1991)

[5] H. Hesham., Thermo acoustic Random Response of Shape Memory Alloy Hybrid Composite Plates, JOURNAL OF AIRCRAFT Vol. 45, No. 3 (2008)

[6] Y. SHI., R.Y.Y. Lee, , and C. MEI., Thermal Post buckling of Composite Plates Using the Finite Element Modal Coordinate Method, Journal of Thermal Stresses, Vol. 22, No,. 6, Aug. (1999), pp. 595-614

[7] R. Jones., and J. Mazumdar., Vibration and Buckling of Plates at Elevated Temperatures, International Journal of Solids and Structures, Vol. 16, No. 1, (1980), pp. 61-70

[9] Y. Lee., R. Y Shi., and C. Mei., Coexisting Thermal Post buckling of Composite Plates with Initial Imperfections Using Finite Element Modal Method, Journal of Thermal Stresses, Vol. 22, No. 6, (1999), pp. 595-614

[10] H. Ibrahim., M. Tawfik., and .M. Al-Ajmi., Thermal Buckling and Nonlinear Flutter Behavior of Functionally Graded Material Panels, Journal of Aircraft, Vol. 44, No. 5, (2007), pp. $1610-1618$

[11] J. E Locke., Finite Element Large Deflection Random Response of Thermally Buckled Plates, Journal of Sound and Vibration, Vol. 160, No. 2, (1993), pp. 301-312.

[12] J. M Dhainaut., B. Duan., C. Mei., S. M. Spottswood., and H.F Wolfe., Nonlinear Response of Composite Panels to Random Excitations at Elevated Temperatures, Structural Dynamics: Recent Advances, Southampton Univ, Southampton, England, U.K, (2000), pp. 769-784 